\title{
Multilevel Programming Reliability in Si-doped GeSbTe for Storage Class Memory
}

\author{
G. Lama*, M. Bernard, N. Bernier, G. Bourgeois, E. Nolot, N. Castellani, J. Garrione, M. C. Cyrille, \\ G. Navarro* and E. Nowak \\ CEA-Leti, Université Grenoble Alpes, F-38000 Grenoble, France \\ * Contact authors: giusy.lama@cea.fr, gabriele.navarro@cea.fr
}

\begin{abstract}
Phase-Change Memory (PCM) demonstrated to be a promising Non-Volatile Memory technology to address Storage Class Memory (SCM) applications that can be distinguished in memory-type and storage-type. In this work we show how $\alpha$ GeSbTe (aGST) alloy can address both SCM types, in particular using Si doping. Thanks to electrical characterization of $4 \mathrm{~kb}$ PCM arrays, supported by TEM analyses, we demonstrate how Si doping in $\alpha$ GST can lead to a huge improvement of MLC operations using a doublepulse protocol. This result, combined with an improved data retention, proves Si-doped aGST suitability for storage-type SCM, whereas high endurance and high speed in undoped aGST allows to target memory-type SCM.
\end{abstract}

\section{Keywords- PCM, SCM, GeSbTe, Si-doping, multilevel}

\section{INTRODUCTION}

Phase-Change Memory (PCM) entered recently in the market with the objective to fill the gap in performances between main memory and storage, concretizing the concept of Storage Class Memory (SCM) [1]. In this revolutionized memory hierarchy, SCM can be closer in terms of performance either to memory (M-SCM) or storage (S-SCM). M-SCM requires speed and endurance, on the contrary, S-SCM must be truly non-volatile (i.e. good data retention) and must provide higher density at a lower cost [2]. In order to improve the density without impact on masks addition and costs, multi-level cell (MLC) capability becomes a key feature. In this paper we investigate Si-doping in $\alpha \mathrm{GeSbTe}(\alpha \mathrm{GST})$ phase-change material to easily tune the crystallization degree in PCM devices, achieving an extremely low variability of intermediate resistance states. TEM analyses are used to support our findings. Therefore, we demonstrate more reliable MLC operations in Si-doped $\alpha \mathrm{GST} 4 \mathrm{~kb}$ arrays applying a simple double-pulse programming protocol. Finally, our results enable $\alpha \mathrm{GST}$ as promising candidate for SCM, making possible to address at the same time M-SCM, thanks to its high SET speed and endurance, and S-SCM, if doped with the right Si content.

\section{DATA/RESULTS AND DISCUSSION}

$\alpha \mathrm{GST}$ and Si-doped $\alpha \mathrm{GST}$ with two different $\mathrm{Si}$ concentration $(\alpha \mathrm{GST}+\mathrm{Si} 1$ and $\alpha \mathrm{GST}+\mathrm{Si} 2$, with respectively lower and higher Si percentage), were integrated in "Wall" PCM $4 \mathrm{~kb}$ arrays, for electrical measurements and statistical analysis, and on blanket wafers for material analyses.

The resistivity as a function of temperature of as-deposited amorphous layers is reported in Fig. 1. Both the resistivity and the crystallization temperature increase as the $\mathrm{Si}$ amount increases, since likely $\mathrm{Si}$ concentrates at the grain boundaries suppressing grain growth and retarding the crystallization process [3]. The resistance-vs-current (R-I) curves (Fig. 2) show that RESET current decreases in Si doped devices, with a reduced variability of R-I characteristic in $\alpha \mathrm{GST}+\mathrm{Si} 2$. However, the Si-doping leads to a lower crystallization (i.e. SET) speed (Fig. 3).

The subthreshold slope $(S T S=d \ln (I) / d V)$ measured in RESET devices, reported in Fig. 4, evidences that in aGST the conduction depends considerably on the temperature, whereas STS remains constant with temperature in Si-doped $\alpha \mathrm{GST}$. STS variation as a function of $(k T)^{-1}$ is proportional to the trap-to-trap distance $\Delta z$ [4] that is higher in $\alpha \mathrm{GST}$ than in $\alpha \mathrm{GST}+\mathrm{Si}$, indicating that $\mathrm{Si}$-doped $\alpha \mathrm{GST}$ contains a higher number of defects introduced by $\mathrm{Si}$, which gives rise to an amorphous structure with a higher disorder and more stable against crystallization.

We analyzed the crystallization process applying a series of short pulses (20 ns) in $\alpha \mathrm{GST}$ and $\alpha \mathrm{GST}+\mathrm{Si} 2$ devices pre-programmed in RESET state (Fig. 5). A gradual expected crystallization is observed in $\alpha$ GST. On the contrary, $\alpha \mathrm{GST}+\mathrm{Si} 2$ resistance decreases and stabilizes at about $10^{5} \Omega$, and only after applying about hundred pulses the resistance starts to decrease again down to the same $\alpha$ GST SET resistance. It confirms that Si retards the crystallization process and brings the advantage of a reliable intermediate state. This behavior is statistically confirmed in $4 \mathrm{~kb}$ arrays using a single squared pulse of $\sim 1 \mu$ s (Fig. 6). Transmission Electron Microscopy (TEM) images of devices programmed in the intermediate state (Fig. 7a) and in SET state (Fig. 7b) evidence that the SET state features a long range crystalline structure, otherwise the intermediate state contains localized crystalline grains embedded in an amorphous matrix. To establish the best protocol to achieve multiple resistive states (MLC) in $\alpha \mathrm{GST}$ and $\alpha \mathrm{GST}+\mathrm{Si} 2$ arrays we studied different programming pulses varying durations and current amplitudes, starting either from SET state (Fig. 8c-d) or from RESET state (Fig. 8a-b, Fig. 9). In $\alpha \mathrm{GST}$, the variability is higher in each test realized, while $\alpha \mathrm{GST}+\mathrm{Si} 2$ shows a low variability when a $1 \mu \mathrm{s}$ long squared pulse is applied on RESET cells (Fig. 8b). An even finer control (Fig. 9c) with low variability (Fig. 9d) of the programmed resistance is obtained in $\alpha \mathrm{GST}+\mathrm{Si} 2$ tuning the pulse fall time. The endurance of the 3 states (SET, RESET and intermediate) is showed to depend on RESET pulse duration (i.e. energy). A low energy $30 \mathrm{~ns}$ RESET pulse allows to reach $10^{5}$ cycles with no degradation of the programmed states (Fig. 10), while a longer RESET pulse of $300 \mathrm{~ns}$, leads to an early degradation of both SET and intermediate states, with a probable Si migration outside of the active region of the device. Endurance tests with different SET/RESET pulses durations (i.e. energy) were realized to extrapolate the maximum endurance achievable with the minimum programming time (Fig. 11) [5]. The failure conditions to calculate the number of cycles take into account a reduction of the RESET resistance and of the reading window, but we added for $\alpha \mathrm{GST}+\mathrm{Si} 2$ the condition on the degradation of the SET state, which is correlated to the loss of the intermediate state (Fig. 10a). Indeed, the endurance in $\alpha \mathrm{GST}+\mathrm{Si} 2$ could be higher than $10^{8}$ (inset in Fig. 11), despite the loss of MLC behavior. We extrapolate a higher endurance for $\alpha \mathrm{GST}$ than in $\alpha \mathrm{GST}+\mathrm{Si} 2$ (MLC).

Furthermore, Si-addition in aGST improves the RESET and the intermediate state retention at $100{ }^{\circ} \mathrm{C}$ as illustrated in Fig. 12, due to the higher crystallization temperature of Si-doped $\alpha \mathrm{GST}$ (Fig. 1).

A multilevel iterative program-and-verify (PV) protocol is proposed in which the programming current $\left(\mathrm{I}_{\mathrm{N}}\right)$ is updated at each iteration, depending on whether the resistances obtained are lower or higher than the target range, and the device pre-programmed in the RESET state (Fig. 13). Already using only a double-pulse operation (RESET + programming) the percentage of devices in the $4 \mathrm{~kb}$ array reaching the target resistance range is higher in $\alpha \mathrm{GST}+\mathrm{Si} 2$ than in $\alpha \mathrm{GST}$. A single PV iteration is not sufficient for $\alpha \mathrm{GST}$ to reach the same $\alpha \mathrm{GST}+\mathrm{Si} 2$ yield, demonstrating the high suitability of $\alpha \mathrm{GST}+\mathrm{Si} 2$ in MLC operations.

\section{CONCLUSIONS}

This work investigates the effects of Si-doping in $\alpha$ GST. In particular, we show an improvement of MLC capability in Si-doped $\alpha$ GST and a better data retention than undoped $\alpha \mathrm{GST}$. These characteristics make $\alpha \mathrm{GST}$ suitable for S-SCM if properly doped with $\mathrm{Si}$, and at the same time M-SCM thanks to the high speed and high endurance of undoped $\alpha \mathrm{GST}$ (Fig. 14).

ACKNOWLEDGEMENTS

This work has been partially supported by the European 783176 WAKeMeUP Project, financed by European commission, French government and Auvergne-Rhône Alpes Region.

REFERENCES

「11 P. Cappelletti, IEDM 2015 Tech. Digest p 10.1. 「21 S. W. Fong et al., IEEE Transactions on Electron Devices, vol. 64, no. 11, pp. 43744385, 2017. [3] Seong-Min Jeong et al., 2009 Jpn. J. Appl. Phys. 48 045503. [4] D. Ielmini and Y. Zhang, J. Appl. Phys., vol. 102, no. 5, p. 054517, Sep. 2007. [5] G. Lama et al., Microelectronics Reliaiblity 2020 (in press). 


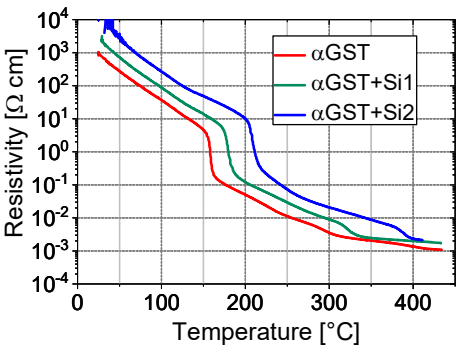

Fig. 1. Resistivity of as-deposited $\alpha \mathrm{GST}, \alpha \mathrm{GST}+\mathrm{Si} 1$, $\alpha \mathrm{GST}+\mathrm{Si} 2$ as a function of temperature measured at a rate of $10^{\circ} \mathrm{C} / \mathrm{min}$.

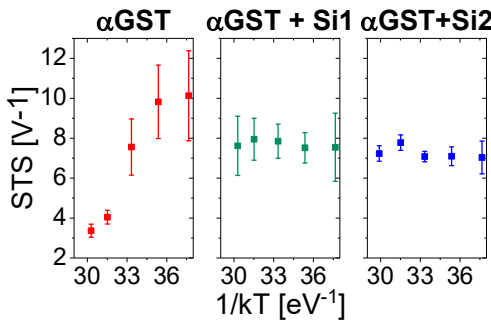

Fig. 4. Subthreshold slope (STS) calculated at different temperatures (4 devices for each composition) is represented as a function of $1 / k T$.
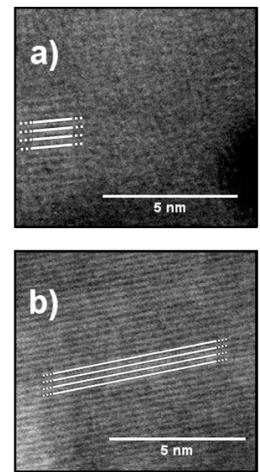

Fig. 7. TEM images performed in the active region of two $\alpha \mathrm{GST}+\mathrm{Si} 2$ devices programmed respectively in the intermediate state (a) and in the SET state (b).

a) $300 \mathrm{~ns}$ RESET width time

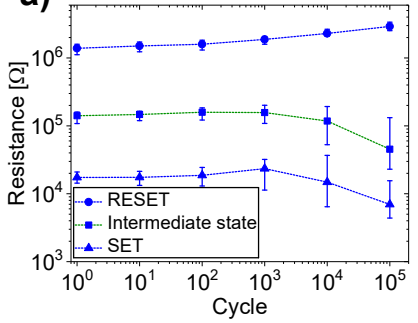

Fig. 10. Endurance test performed in $\alpha \mathrm{GST}+\mathrm{Si} 2$ applying a RESET pulse with a width time of $300 \mathrm{~ns}$ (a, top graph) and $30 \mathrm{~ns}$ (b, bottom graph) on a population of about 100 devices.

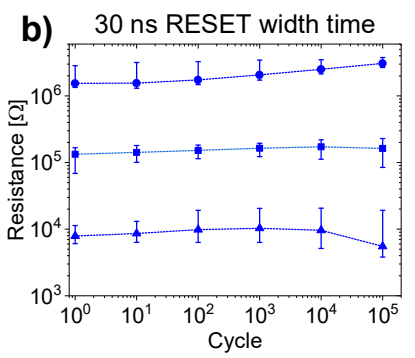

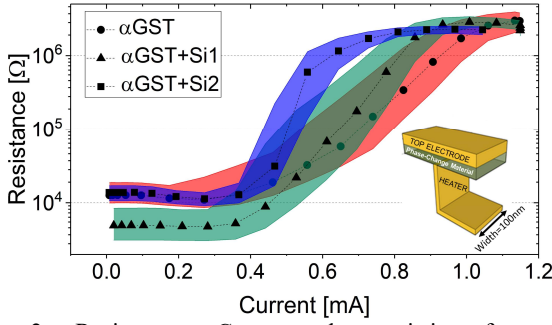

Fig. 2. Resistance-vs-Current characteristics for $\alpha \mathrm{GST}$, $\alpha \mathrm{GST}+\mathrm{Si1}, \alpha \mathrm{GST}+\mathrm{Si} 2$ based PCMs: median, 16th and 84th percentiles (corresponding to $1 \sigma$ ) of the resistance values obtained in $4 \mathrm{~kb}$ arrays are represented. On the bottom right is reported the scheme of a typical "Wall" PCM device used in this work.

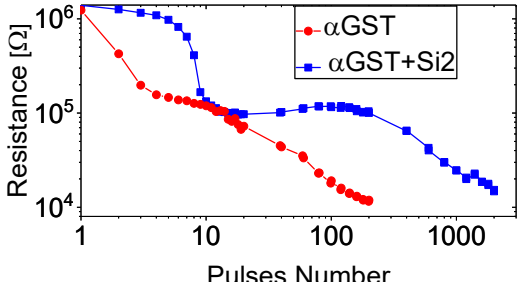

Fig. 5. Resistance achieved in $\alpha \mathrm{GST}$ and $\alpha \mathrm{GST}+\mathrm{Si} 2$ devices, starting from a RESET state, applying consecutively a sequence of short pulses with $10 \mathrm{~ns}$ rise/fall time and $20 \mathrm{~ns}$ width time.

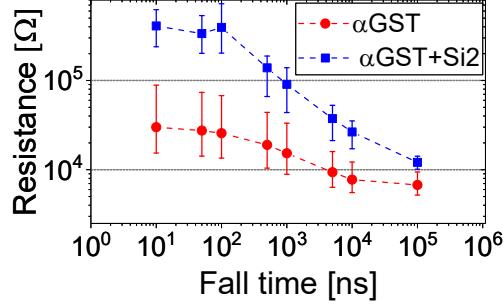

Fig. 3. SET programming speed test performed in $4 \mathrm{~kb}$ arrays with constant pulse width $(300 \mathrm{~ns})$ and increasing pulse fall time: median, 16th and 84th percentiles of the resistance values are represented.

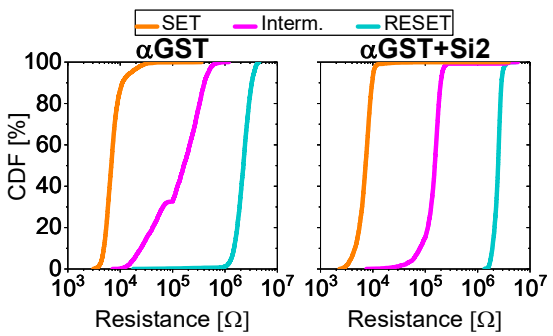

Fig. 6. Cumulative distributions of resistances measured in $\alpha \mathrm{GST}$ and $\alpha \mathrm{GST}+\mathrm{Si} 24 \mathrm{~kb}$ arrays. The intermediate state is achieved with a current of $0.2 \mathrm{~mA}$ and a long width time pulse $(\sim 1 \mu \mathrm{s})$.
Starting from RESET Starting from SET

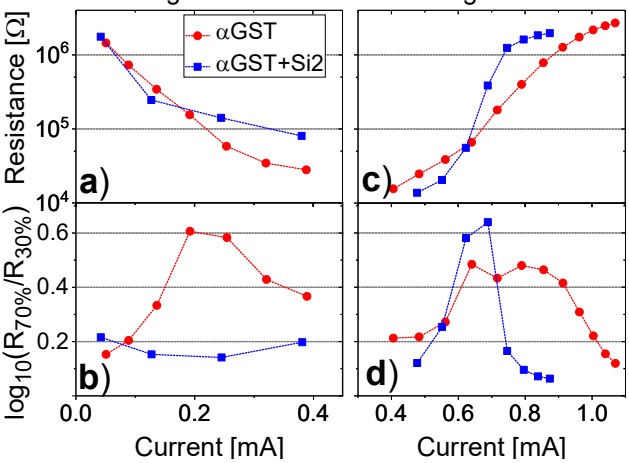

$10 \mathrm{~ns}$ pulse fall time $\quad 10 \mu \mathrm{s}$ pulse fall time

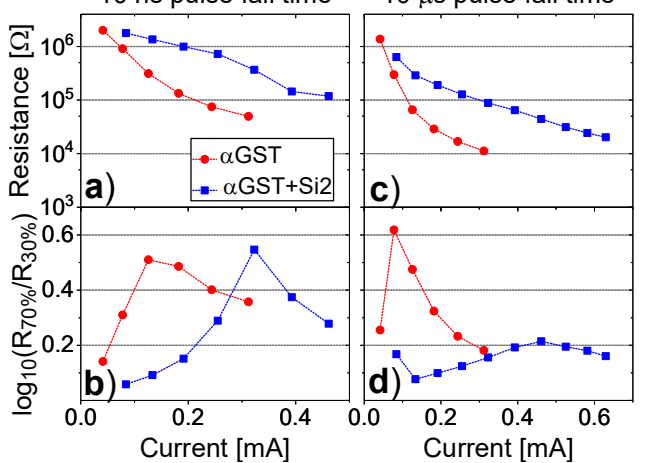

Fig. 8. Intermediate states achieved starting from RESET state $(a, b)$ with $1 \mu \mathrm{s} \quad$ Fig. 9. Intermediate states achieved starting from RESET (a,b) with square pulses and from SET state (c,d) with $300 \mathrm{~ns}$ square pulses. Median $(\mathrm{a}, \mathrm{c})$ and variability $(b, d)$ of the resistances in $4 \mathrm{~kb}$ arrays are represented.

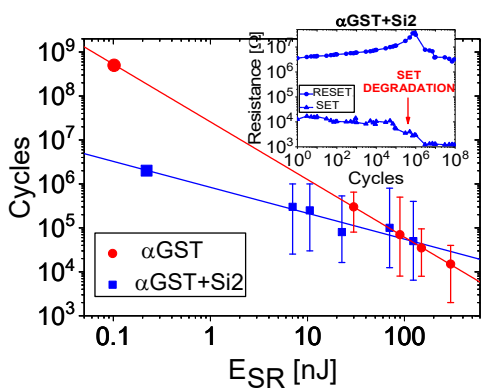

Fig. 11. Number of cycles achieved for different SET+RESET pulses duration (i.e. energy) in $\alpha \mathrm{GST}$ and $\alpha \mathrm{GST}+\mathrm{Si} 2$ devices pllowing to extrapolate a higher endurance in $\alpha$ GST. Each condition was applied on a population of about 40 devices. Median and $1 \sigma$ are represented. In the inset, an endurance test on a $\alpha \mathrm{GST}+\mathrm{Si} 2$ device.
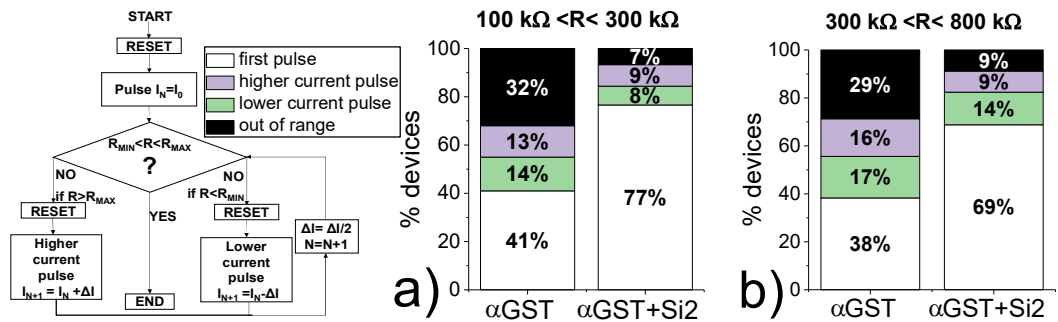

Fig. 13. Program-and-Verify (PV) protocol used to get intermediate resistive states in $\alpha \mathrm{GST}$ and $\alpha \mathrm{GST}+\mathrm{Si} 2$ $4 \mathrm{~kb}$ arrays. The target intermediate resistance range is reported above the graphs a) and b). In white, the percentage of the devices getting the target after the first programming pulse, in purple and in green the devices getting the target after a single-step of PV and in black those remaining out of the range. is $300 \mathrm{~ns}$ for both. Median (a,c) and variability (b,d) of the resistances in $4 \mathrm{~kb}$ arrays are represented.

$\alpha$ GST $\quad \alpha$ GST+Si1

$\alpha \mathrm{GST}+\mathrm{Si} 2$

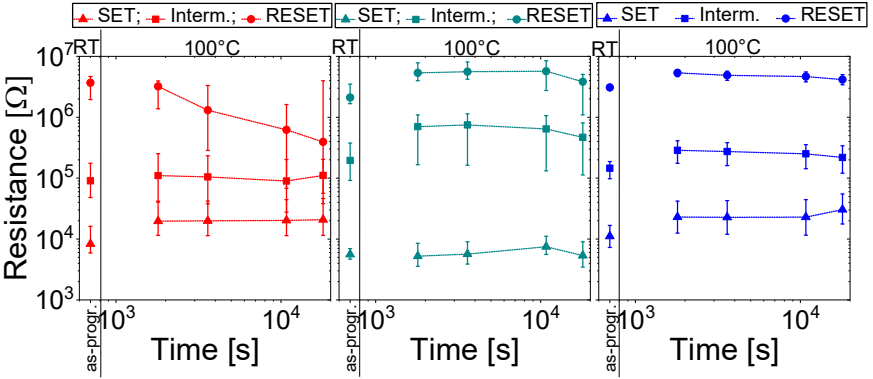

Fig. 12. Data retention at $100^{\circ} \mathrm{C}$ of SET, RESET and an intermediate state for the three compositions measured in $4 \mathrm{~kb}$ arrays. Median and $1 \sigma$ are reported after programming as.-progr.) at room temperature (RT) and along time after annealing at $100^{\circ} \mathrm{C}$.

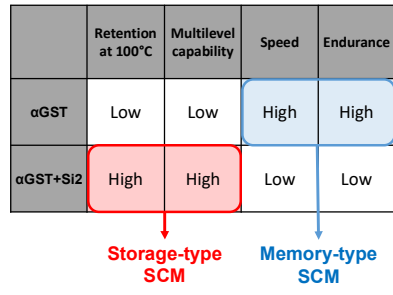

Fig. 14. Summary of the features achieved in $\alpha \mathrm{GST}$ and $\alpha \mathrm{GST}+\mathrm{Si} 2$ based PCM devices, showing the possibility to target both S-SCM and M-SCM specifications. 